This is a postprint version of the following published document:

N'souglo, K.E.; Rodríguez-Martínez, J.A. Non-uniform distributions of initial porosity in metallic materials affect the growth rate of necking instabilities in flat tensile samples subjected to dynamic loading, in: Mechanics Research Communications, 91, pp. 87-92, July 2018

DOI: https://doi.org/10.1016/j.mechrescom.2018.05.004

(C) 2018 Elsevier Ltd. All rights reserved.

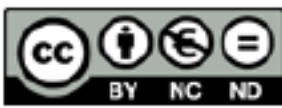

This work is licensed under a Creative Commons Attribution-NonCommercialNoDerivatives 4.0 International License. 


\title{
Non-uniform distributions of initial porosity in metallic materials affect the growth rate of necking instabilities in flat tensile samples subjected to dynamic loading
}

\author{
K. E. N’souglo, J. A. Rodríguez-Martínez* \\ Department of Continuum Mechanics and Structural Analysis. University Carlos III of Madrid. Avda. de la Universidad, 30.28911 Leganés, Madrid, Spain
}

\begin{abstract}
In this paper we assess, using finite element calculations performed with ABAQUS/Explicit, the influence of porosity in the development of necking instabilities in flat metallic samples subjected to dynamic tension. The mechanical behaviour of the material is described with the Gurson-Tvergaard-Needleman $[6,22,23]$ constitutive model pre-implemented in the finite element code. The novelty of our methodology is that we have included in the gauge of the specimen various non-uniform distributions of initial porosity which, in all cases, keep constant the average porosity in the whole sample. This has been carried out assigning random values of initial porosity (within specified bounds) to some nodes and zero to the others. Therefore, the larger the percentage of nodes with non-zero initial porosity, the smaller their initial value of porosity. The goal is to provide an idealized modelling of the distributions of void nucleating particles which in many structural metals nucleate early in the deformation process and lead to material porosity. The key point of this paper is that, following this methodology, we reproduce the experimentally-observed asymmetric-growth of the pair of necking bands which define the localization process in flat tensile samples subjected to dynamic loading [25].
\end{abstract}

Keywords:

Dynamic tension, Necking instabilities, Porous plasticity, Finite element simulations

\section{Introduction}

The study of dynamic necking instabilities which trigger failure of metallic (ductile) samples subjected to impact tensile loading has focussed the efforts of many researchers over the last 80 years. The experimental works of Mann [12, 13] in the 30s, and Clark and co-workers $[4,3]$ in the $40 \mathrm{~s}$, were among the first papers in this topic. These authors performed tension tests using cylindrical specimens of different materials for impact velocities up to $100 \mathrm{~m} / \mathrm{s}$, and showed that the location of the neck in the sample, and thus the final fracture, was dependent on the applied velocity. This correlation between necking/fracture location and impact velocity was rationalized using the theory of plastic strain propagation that had just been developed by Von-Kármán [26]. It was concluded that the intervention of stress waves in the sample, generated due to the application of a sudden impact to a specimen at rest, yields severe strain gradients in the sample (spatial and temporal) which determine the position where the fracture occurs. Post-mortem examination of the specimens revealed considerable ductility inside the neck for all materials and velocities that these authors tested, the final fracture showing a deep cup and cone which were more acute as the impact velocity was increased. Similar experimental observations have been reported in many other studies in which metallic cylindrical bars were tested dynamically, see for instance the recent papers of Rittel and co-workers [17, 18].

${ }^{*}$ Corresponding author. Tel. +34 916249904; Fax: +34 916249430. Email address: jarmarti@ing.uc3m.es
Since the 90 s of previous century, using flat samples instead of round bars in the dynamic tensile testing of metallic materials has become a common practice. The main reason is the interest of the automotive industry in the mechanical characterization of metallic sheets used to build crashworthiness structures [2]. Using high speed servo-hydraulic machines, experiments in metallic sheets can be performed for velocities up to $20 \mathrm{~m} / \mathrm{s}$, and strain rates up to $10^{3} \mathrm{~s}^{-1}$. The failure of the sample is preceded by the formation of a pair of necking bands which are aligned with the two directions of zero extension contained in the plane of the specimen [7]. One of these bands grows faster than the other, leading to the formation of a single crack inclined $54.7^{\circ}$ (if the material is isotropic) with respect to the loading direction.

Nevertheless, to the authors' knowledge, most of the attempts carried out so far to simulate numerically the tensile behaviour of flat samples subjected to dynamic loading predict that, contrary to the experimental observations, the growth rate of the pair of necking bands which form the localization process is largely similar [11, 16, 15, 9, 25]. It seems that, if the material is isotropic and homogeneous, and the boundary conditions ensure that the stress state in the sample is uniaxial until localization starts, there is not a perturbation in the numerical model that could lead to the asymmetric growth of the two bands. The mechanical perturbations coming from the propagation of waves within the specimen, and the numerical perturbations coming from the discretization of the work piece do not seem to produce an important difference in the rate of development of the necking bands. However, in some works, geometric perturbations have been 
included in the specimen model to trigger the asymetric grow rate of the necking bands [14]. In this paper we propose a different approach, and develop a simple finite element model which, including non-uniform distributions of porosity in the specimen material, allows to reproduce the experimentallyobserved asymmetric-growth of the pair of necking bands which define the localization process in flat tensile samples subjected to dynamic loading.

\section{Constitutive framework}

The mechanical behaviour of the material is described using the Gurson-Tvergaard-Needleman (GTN) constitutive model [6, 22, 23] pre-implemented in ABAQUS/Explicit [20]. For the sake of clarity, the main features of the model are briefly presented in this section.

The flow potential has the form:

$$
\Phi=\left(\frac{\sigma_{e}}{\sigma_{y}}\right)^{2}+2 q_{1} f^{*} \cosh \left(\frac{3 q_{2} \sigma_{h}}{2 \sigma_{y}}\right)-1-\left(q_{1} f^{*}\right)^{2}
$$

where the effective Mises stress, $\sigma_{e}$, and the hydrostatic pressure, $\sigma_{h}$, are defined by:

$$
\sigma_{e}=\sqrt{\frac{3}{2} s: s} ; \quad \sigma_{h}=\frac{1}{3} \boldsymbol{\sigma}: \mathbf{1} ; \quad \boldsymbol{s}=\boldsymbol{\sigma}-\sigma_{h}: \mathbf{1}
$$

where $\sigma$ is the macroscopic Cauchy stress tensor, $s$ is its deviatoric part, and $\mathbf{1}$ is the unit second order tensor.

Moreover, $\sigma_{y}$ is the flow strength of the fully dense matrix material described in the present work by the following power-type relation [21]:

$$
\sigma_{y}=\Psi\left(\bar{\varepsilon}^{p}, \dot{\bar{\varepsilon}}^{p}\right)=\sigma_{0}\left(1+\frac{\bar{\varepsilon}^{p}}{\varepsilon_{0}}\right)^{n}\left(\frac{\dot{\bar{\varepsilon}}^{p}}{\dot{\varepsilon}_{0}}\right)^{m}
$$

where $\bar{\varepsilon}^{p}=\int_{0}^{t} \dot{\bar{\varepsilon}}^{p}(\tau) d \tau$ and $\dot{\bar{\varepsilon}}^{p}$ are the effective plastic strain and the effective plastic strain rate in the matrix material, respectively. Moreover, $\sigma_{0}, n$ and $m$ are material parameters, and $\varepsilon_{0}$ and $\dot{\varepsilon}_{0}$ are the reference strain and strain rate, respectively. Note that, for the sake of simplicity, the temperature dependence of the flow strength is not considered.

In Eq. (1), $q_{1}$ and $q_{2}$ are material parameters, and the function $f^{*}=f^{*}(f)$, where $f$ is the void volume fraction, is given by:

$$
f^{*}= \begin{cases}f & \text { if } f<f_{c} \\ f_{c}+\frac{\left(f_{u}-f_{c}\right)\left(f-f_{c}\right)}{\left(f_{f}-f_{c}\right)} & \text { if } f_{c} \leqslant f \leqslant f_{f} \\ f_{u} & \text { if } f>f_{u}\end{cases}
$$

where $f_{c}$ is the void volume fraction at which voids coalesce, $f_{f}$ is the void volume fraction at final fracture of the material and $f_{u}=1 / q_{1}$ is the ultimate void volume fraction.

The rate of deformation tensor is taken to be the sum of an elastic part, $\boldsymbol{d}^{e}$, and a plastic part, $\boldsymbol{d}^{p}$, as follows:

$$
\boldsymbol{d}=\boldsymbol{d}^{e}+\boldsymbol{d}^{p}
$$

where the elastic part is related to the rate of the stress by the following hypo-elastic law:

$$
\dot{\sigma}=C: d^{e}=C:\left(d-d^{p}\right)
$$

with $\dot{\sigma}$ being the objective stress rate (it corresponds to the Green-Naghdi derivative in ABAQUS/Explicit [20]) and $\boldsymbol{C}$ being the tensor of isotropic elastic moduli given by:

$$
C=2 G I^{\prime}+K \mathbf{1} \otimes \mathbf{1}
$$

where $G$ is the elastic shear modulus, $K$ is the bulk modulus and $\boldsymbol{I}^{\prime}$ is the unit deviatoric fourth order tensor.

The plastic part of the rate of deformation tensor follows the direction normal to the flow potential:

$$
\boldsymbol{d}^{p}=\dot{\lambda} \frac{\partial \Phi}{\partial \boldsymbol{\sigma}}
$$
factor.

where $\dot{\lambda}$ is the non-negative plastic flow proportionality

The plastic part of the rate of deformation tensor and the effective plastic strain rate in the matrix material are related by enforcing equality between the rates of macroscopic and matrix plastic work:

$$
\boldsymbol{\sigma}: \boldsymbol{d}^{p}=(1-f) \sigma_{y} \dot{\bar{\varepsilon}}^{p}
$$

Moreover, assuming the incompressibility of the matrix material, the evolution of the void volume fraction is defined as:

$$
\dot{f}=(1-f) \mathbf{d}^{p}: \mathbf{1}
$$

Note that void nucleation is not considered in the present analysis. Hence, if the initial void volume fraction is zero, the macroscopic material is fully dense and follows Mises plasticity.

In ABAQUS/Explicit, the integration of the constitutive model relies on the consistency condition during plastic loading:

$$
\dot{\Phi}=0
$$

Details regarding the integration of the constitutive model are not given herein but can be found in [1].

The flow strength of the matrix material, Eq (3), has been used along with the GTN model pre-implemented in ABAQUS/Explicit through a user-defined subroutine VUHARD [20]. The material parameters related to the flow potential, Eq. (1), and the flow strength of the matrix material, Eq. (3), taken from [21], are given in Table 1. The av- 


\begin{tabular}{lll}
\hline Symbol & Property and units & Value \\
\hline$\rho_{0}$ & Initial density $\left(\mathrm{kg} / \mathrm{m}^{3}\right)$ & 7600 \\
\hline$G$ & Elastic shear modulus $(\mathrm{GPa})$, Eq. (7) & 26.9 \\
$K$ & Bulk modulus $(\mathrm{GPa})$, Eq. (7) & 58.3 \\
\hline$q_{1}$ & Material parameter, Eq. (1) & 1.25 \\
$q_{2}$ & Material parameter, Eq. (1) & 1.0 \\
$\sigma_{0}$ & Reference yield stress ( $\mathrm{MPa})$, Eq. (3) & 300 \\
$n$ & Strain hardening sensitivity, Eq. (3) & 0.1 \\
$m$ & Strain rate sensitivity, Eq. (3) & 0.01 \\
$\varepsilon_{0}$ & Reference strain, Eq. (3) & 0.00429 \\
$\dot{\varepsilon}_{0}$ & Reference strain rate ( $\left.\mathrm{s}^{-1}\right)$, Eq. (3) & 1000 \\
\hline$f_{0}$ & Average value of initial VVF & 0.01 \\
$f_{c}$ & VVF at which voids coalesce, Eq. (4) & 0.12 \\
$f_{f}$ & VVF at final fracture, Eq. (4) & 0.25 \\
$f_{u}$ & Ultimate VVF, Eq. (4) & 0.8 \\
\hline
\end{tabular}

Table 1: Material parameters used in the finite element calculations [21]. $V V F$ stands for void volume fraction (porosity).

erage value of initial void volume fraction, from now on indistinctly referred to as porosity, is $f_{0}=0.01$ (average value over the whole gauge of the sample, see section 3.2).

\section{Finite element model}

3D finite element simulations of flat tensile samples subjected to dynamic tension are carried out using the comercial finite element code ABAQUS/Explicit [20].

\subsection{Dynamic tensile specimen}

The geometry and dimensions of the specimens simulated, taken from [25], are shown in Fig. 1. The initial and boundary conditions of the problem are:

- Initial conditions: the specimen is initially undeformed, unstressed and at rest.

$$
\begin{aligned}
& V_{i}\left(X_{1}, X_{2}, X_{3}, 0\right)=0 \\
& \sigma_{e}\left(X_{1}, X_{2}, X_{3}, 0\right)=0 ; \quad \bar{\varepsilon}^{p}\left(X_{1}, X_{2}, X_{3}, 0\right)=0
\end{aligned}
$$

where $V_{i}$, with $i=1,2,3$, are the material particle velocities along the $X_{1}, X_{2}$ and $X_{3}$ directions, respectively. Moreover, recall that $\sigma_{e}$ is the effective Mises stress and $\bar{\varepsilon}^{p}$ is the effective plastic strain in the matrix material.

- Boundary conditions: one end of the specimen is clamped while the other is subjected to a Heaviside step velocity boundary condition.

$$
V_{1}\left(L_{0}+2 L_{1}, X_{2}, X_{3}, t\right)=V ; \quad U_{i}\left(0, X_{2}, X_{3}, t\right)=0
$$

where $U_{i}$, with $i=1,2,3$, are the material particle displacements along the $X_{1}, X_{2}$ and $X_{3}$ directions, respectively. All other faces are traction free.

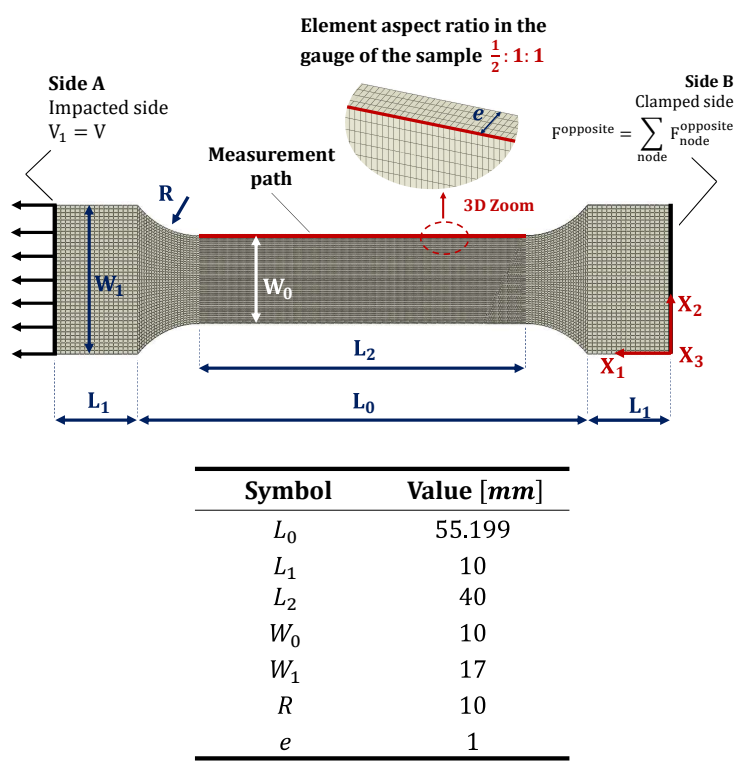

Figure 1: Finite element model. Mesh, dimensions and boundary conditions.

The samples are meshed with 63680 eight-node tri-linear brick elements with reduced integration (C3D8R in ABAQUS notation). The elements in the gauge have an initial aspect ratio $\frac{1}{2}: 1: 1$ (with dimensions $0.125 \times 0.25 \times 0.25 \mathrm{~mm}^{3}$ ). The integral viscoelastic approach available in ABAQUS/Explicit [20] has been used to prevent hourglass deformation modes, with the scale factor used for all hourglass stiffnesses being equal to one.

\subsection{Porosity distribution}

Void nucleating particles (idealized here as initial material porosity) are present in many metallic materials, and they are considered as sources of damage development and preferential sites for plastic localization and inception of cracks. These defects, which are usually non-uniformly distributed in the bulk, try to be replicated in this paper using a simple methodology specifically devised for the purpose.

Firstly, we set an average value of initial porosity in the gauge of the specimen, $f_{0}$. The value of $f_{0}$ is 0.01 in all the calculations of section 4 . We only pay attention to the gauge because only this part of the sample deforms during the loading process. Hence, the specimen material outside the gauge is taken as fully dense. The average value of the initial porosity in the gauge is obtained assigning finite (positive) values of initial porosity only to some nodes (and zero to the others). The percentage of nodes, over the total number of nodes in the gauge, that do have initial porosity is denoted as $P$. Several values of $P$, ranging from $10 \%$ to $25 \%$, have been investigated. As $P$ increases, more nodes have an initial (positive) value of porosity. The specific nodes with positive initial porosity are selected randomly using the function randperm of MATLAB. Each of these nodes has allotted a random value of initial porosity that was generated using the normal distribution function normrnd (see [24]) which is also pre-implemented in MATLAB. The mean of this distribution is $\mu=f_{0} / P$ and the standard deviation is $\sigma=\mu C_{v}$, where the relative standard deviation, $C_{v}$, determines the maximum 


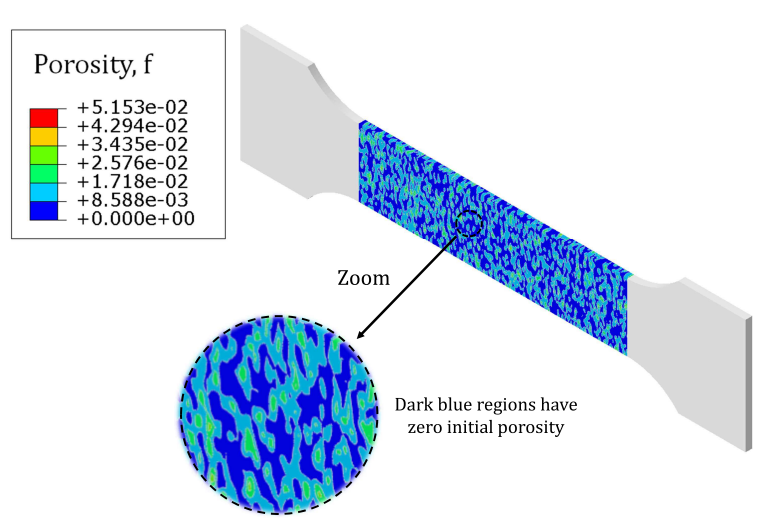

Figure 2: Finite element model. Contours of initial porosity in the gauge of the specimen. The values of $f_{0}, P$ and $C_{v}$ are $0.01,15 \%$ and $15 \%$, respectively.

dispersion of the generated random values of porosity. The value of $C_{v}$ is $15 \%$ in all the calculations of section 4 . Note that, for a given value of $f_{0}$, the mean $\mu$ decreases as $P$ increases. If $P=100 \%$ then $\mu=f_{0}$. Moreover, note that, for any given combination of $P, C_{v}$ and $f_{0}$, we could generate an infinite number of random porosity distributions: different nodes with positive initial porosity and different values of porosity for each one. Nevertheless, assessing the specific effect that the randomness of the porosity distribution has on the finite element results is beyond the scope of this work. Only one random distribution of porosity is used for each value of $P$ studied. We expect the results for the distributions we have analyzed to be representative of other random distributions of porosity.

Fig. 2 shows the contours of initial porosity in the gauge of the specimen for the porosity distribution corresponding to $P=15 \%$. Note that the colour coding corresponds to the values of initial porosity at the material points. The porosity at the material points is calculated by the finite element code interpolating the values that we specify at the nodes. Note that, in ABAQUS/Explicit, even though the porosity is an elementbased variable, it has to be specified at the nodes in order to achieve the discontinuity in the porosity field.

We acknowledge that there are more sophisticated approaches to model the distributions of void nucleating particles which lead to material porosity in structural metals. Nevertheless, we hold that the methodology presented in this section allows for bringing to light interesting features about the influence of the porosity distribution in the development of dynamic necking instabilities.

\section{Results and discussion}

We conducted numerical simulations for various impact velocities between $8 \mathrm{~m} / \mathrm{s}$ and $25 \mathrm{~m} / \mathrm{s}$, which correspond to strain rates between 200 and $625 s^{-1}$. This range of velocities, and strain rates, is typically attained in dynamic tension tests performed in fast servo-hydraulic machines [25]. Note that for this range of velocities, and for this specimen size, inertial effects are less important than in the other types of uniaxial tenstion tests like the rapid expansion of rings, where the applied velocities can reach $200 \mathrm{~m} / \mathrm{s}$, and the strain rates

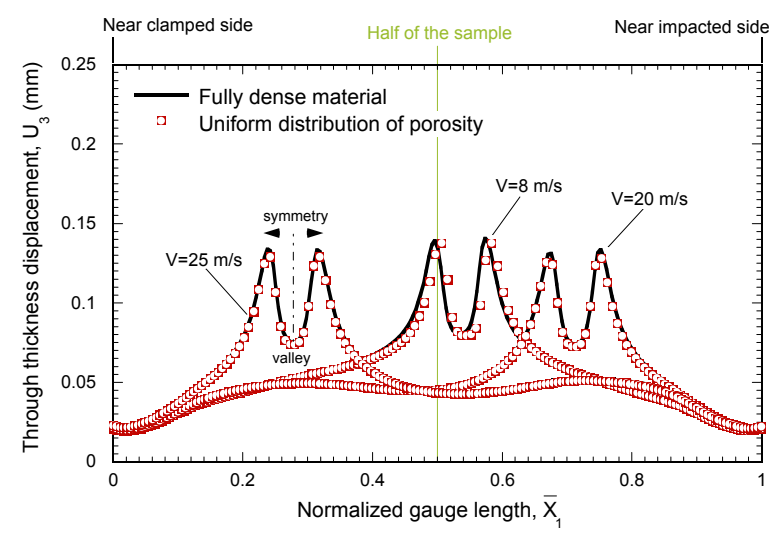

Figure 3: Through-thickness displacement of the edge of the gauge $U_{3}$ versus normalized gauge length $\bar{X}_{1}=\frac{2 X_{1}-2 L_{1}-L_{0}+L_{2}}{2 L_{2}}$ (see Fig. 3). The loading time is such that the maximum value of $\bar{\varepsilon}^{p}$ in the specimen is 1.2. Three different applied velocities are considered: $V=8,20$ and $25 \mathrm{~m} / \mathrm{s}$. Results are presented for the material with uniform distribution of porosity, and the fully dense material.

$5 \cdot 10^{4} s^{-1}[5,28,10]$. In addition to the simulations carried out with the non-uniform distributions of porosity described in previous section $(10 \% \leq P \leq 25 \%)$, we have run calculations with a uniform distribution of porosity $\left(\mu=f_{0}\right.$ and $\left.C_{v}=0\right)$, and with the fully dense material $\left(f_{0}=0\right)$. We start the analysis presenting results obtained for these last two cases.

Fig. 3 shows the through-thickness displacement of the edge of the gauge $U_{3}$ versus the normalized gauge length of the sample $\bar{X}_{1}=\frac{2 X_{1}-2 L_{1}-L_{0}+L_{2}}{2 L_{2}}$ (see Fig. 3), for the material with uniform distribution of porosity, and the fully dense material. Three impact velocities are considered, $V=8,20$ and $25 \mathrm{~m} / \mathrm{s}$, respectively. For each impact velocity, the loading time is such that the maximum value of the equivalent plastic strain in the specimen is $\bar{\varepsilon}^{p}=1.2$. The excursions in displacement represent the pair of necking bands oriented with the directions of zero extension that form the localization pattern in ductile metallic sheets subjected to uniaxial tension [7]. The necking bands are clearly observed in Fig. 4, which shows contours of equivalent plastic strain, for the fully dense material, for the same velocities and loading times of Fig. 3. Note that the location of the necking bands depends on the applied velocity, as shown experimentally and numerically in several publications, e.g. $[19,25]$. Note also that the results obtained for the material with uniform distribution of porosity and the fully dense material are very similar. Including an homogeneous distribution of porosity in the model does not affect significantly the localization process for the impact velocities and material behaviour investigated in this paper. The two excursions of through-thickness displacement which represent the necking bands are largely symmetric with respect to the central point of the valley that they have in between: the two necking bands virtually have the same width and rate of growth. Next, we show that including a non-uniform distribution of porosity in the gauge breaks this symmetry and the two necking bands develop at different speeds, in agreement with the experimental evidences [25].

Fig. 5 shows the through-thickness displacement of the 
Fully dense material

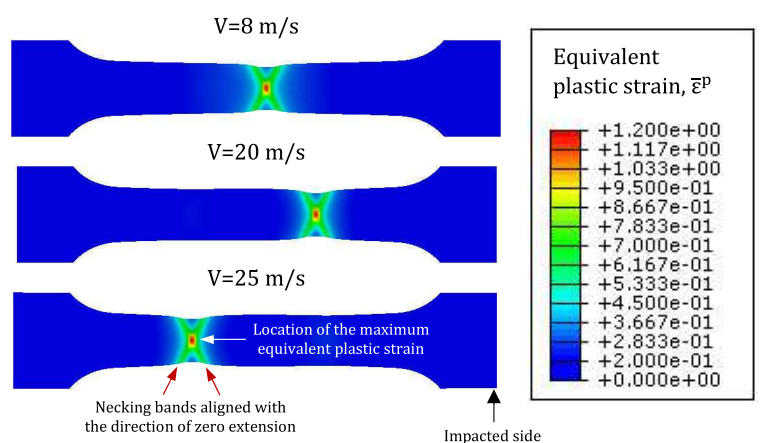

Figure 4: Contours of equivalent plastic strain $\bar{\varepsilon}^{p}$. The loading time is such that the maximum value of $\bar{\varepsilon}^{p}$ is 1.2 . Three different applied velocities are considered: $V=8,20$ and $25 \mathrm{~m} / \mathrm{s}$. Results are presented for the fully dense material.

edge of the gauge $U_{3}$ versus the normalized gauge length of the sample $\bar{X}_{1}$ (see Fig. 3), for the material with uniform distribution of porosity, and the materials with non-uniform distributions of porosity corresponding to $P=10 \%$ and $P=$ $25 \%$. As mentioned before, we have run calculations with other values of $P$ but the results are not shown for the sake of brevity. Three impact velocities are considered: $8 \mathrm{~m} / \mathrm{s}$ in Fig. 5(a), $20 \mathrm{~m} / \mathrm{s}$ in Fig. 5(b), and $25 \mathrm{~m} / \mathrm{s}$ in Fig. 5(c). In all cases the loading time is such that the maximum value of $\bar{\varepsilon}^{p}$ in the specimen is 1.2. Fig. 6 shows contours of equivalent plastic strain $\bar{\varepsilon}^{p}$ for the same uniform and non-uniform distributions of porosity considered in Fig. 5, and for the same loading times and impact velocities: $8 \mathrm{~m} / \mathrm{s}$ in Fig. 6(a), $20 \mathrm{~m} / \mathrm{s}$ in Fig. 6(b), and $25 \mathrm{~m} / \mathrm{s}$ in Fig. 6(c). The non-uniform distributions of porosity play a role in: (1) the location of the gauge where the flow localization occurs and (2) the rate of growth of the necking bands that form the localization pattern.

1. For $V=8 \mathrm{~m} / \mathrm{s}$ the necking bands develop roughly in the center of the sample for the uniform distribution of porosity, closer to the impacted side for $P=10 \%$, and closer to the clamped side for $P=25 \%$. However, for $20 \mathrm{~m} / \mathrm{s}$ and $25 \mathrm{~m} / \mathrm{s}$ the location of the plastic localization becomes largely independent of $P$. In our calculations there is a threshold velocity, $V \approx 14 \mathrm{~m} / \mathrm{s}$, for which the location of flow localization turns from being controlled by the porosity distribution to being controlled by the intervention of wave propagation within the specimen. As the impact velocity increases, the role of the stress waves in the specimen deformation behaviour becomes more important, which leads to a severe heterogeneity in the stress and strain fields in the sample, which eventually dictates the location of the flow localization [27, 8]. We have checked that the threshold velocity depends, for instance, on the average value of porosity in the gauge.

2. Irrespective of the impact velocity, both non-uniform distributions of porosity, $P=10 \%$ and $P=25 \%$, break the symmetry of the pair of necking bands that form the localization pattern. One of the two bands grows faster than the other. This result is in agreement with the experimental evidences reported elsewhere [25]. For in- stance, Fig. 7 shows AISI 430 steel samples, tested by Vaz-Romero et al. [25], with a fracture triggered by a necking band aligned with one of the two directions of zero extension that exist in the loading plane of the specimen. In these experiments the direction of zero extension that leads to fracture was dependent on the applied velocity, as in our calculations. In the simulations of Figs. 5 and 6 , for $V=8 \mathrm{~m} / \mathrm{s}$, the band that grows faster is the one on the left if $P=10 \%$ and the one on the right if $P=25 \%$. For $V=20 \mathrm{~m} / \mathrm{s}$ the band that grows faster in the one on the right, and for $V=25 \mathrm{~m} / \mathrm{s}$ is the one on the left. In our simulations there is a threshold velocity, $V \approx 14 \mathrm{~m} / \mathrm{s}$, for which the band that develops more rapidly becomes independent of the non-uniform distribution of porosity $P$ (for the values of $P$ considered in this work).

However, the non-uniform distributions of porosity do not play a meaningful role in the response of the sample before plastic flow localizes. Note in Fig. 5 that outside the localization area the $U_{3}-\bar{X}_{1}$ curves are very similar for the material with uniform distribution of porosity, and the materials with non-uniform distributions of porosity. For $P=10 \%$ and $P=25 \%$ there are slight oscillations in $U_{3}$ due to the heterogeneity in the porosity level, but the results are virtually overlapped with those obtained for the material with uniform distribution of porosity. Fig. 8 shows the axial force in the clamped side of the specimen $F$ as a function of the loading time $t$ for an impact velocity of $8 \mathrm{~m} / \mathrm{s}$. The force is virtually identical for the material with uniform distribution of porosity and the materials with $P=10 \%$ and $P=25 \%$ until the loading time is $\approx 1000 \mu \mathrm{s}$. This loading time, for which the force starts to drop quickly, determines the onset of plastic localization. Note that the fastest force drop, i.e. the fastest localization process, occurs for $P=10 \%$, and the slowest for the material with homogeneous distribution of porosity. It becomes apparent that, in our calculations, the heterogeneity of the porosity distribution plays a key role in the development of the localization process.

\section{Concluding remarks}

In this paper we have investigated the influence of porosity in the localization of plastic flow in flat tensile samples subjected to dynamic loading. For that purpose we have developed a simple numerical methodology in which non-uniform distributions of porosity are included in the gauge of the specimens simulated. The goal is to provide an idealized modelling of the distributions of void nucleating particles which in many commercially-available metals and alloys nucleate early in the deformation process and lead to material porosity. A key point of the methodology is that, for all the nonuniform distributions of porosity tested, the average porosity in the whole gauge of the specimens was kept constant. The numerical simulations reveal that, because the porosity is not homogeneously distributed in the material, the localization pattern in the sample is formed by a pair of necking bands which grow at different speeds, in agreement with experimental evidences reported in the literature. The calculations also 


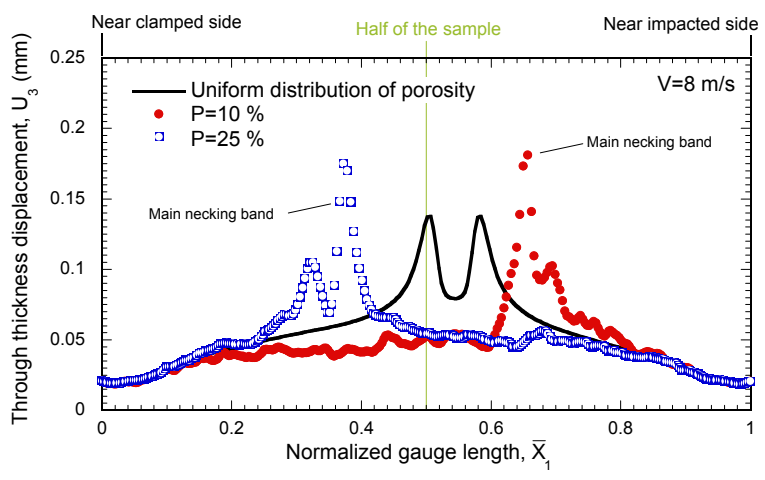

(a)

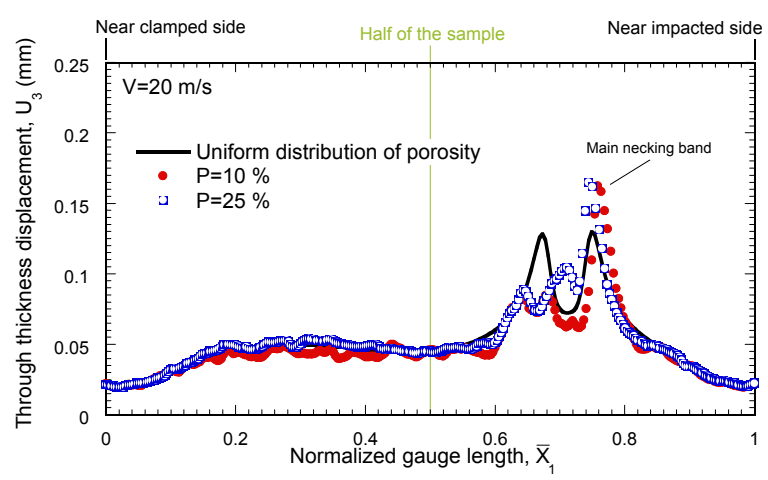

(b)

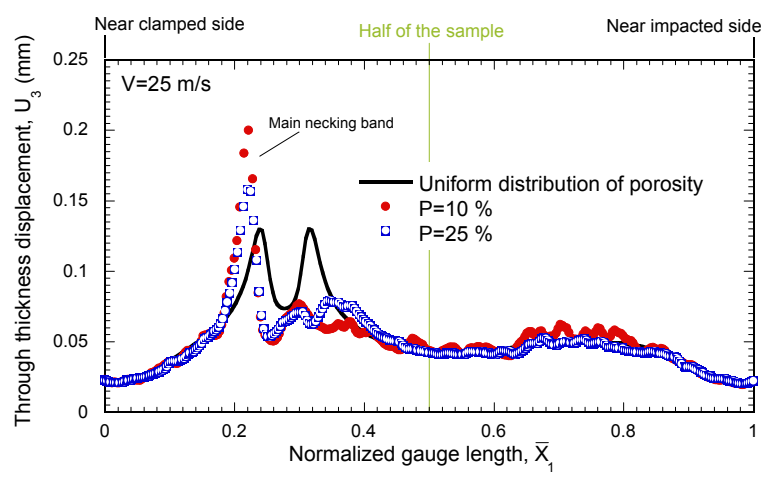

(c)

Figure 5: Through-thickness displacement of the edge of the gauge $U_{3}$ versus normalized gauge length of the sample $\bar{X}_{1}=\frac{2 X_{1}-2 L_{1}-L_{0}+L_{2}}{2 L_{2}}$ for the material with uniform distribution of porosity, and the materials with non-uniform distributions of porosity corresponding to $P=10 \%$ and $P=25 \%$. Three impact velocities are considered: $8 \mathrm{~m} / \mathrm{s}$ in Fig. 5(a), $20 \mathrm{~m} / \mathrm{s}$ in Fig. 5(b), and $25 \mathrm{~m} / \mathrm{s}$ in Fig. 5(c). In all cases the loading time is such that the maximum value of $\bar{\varepsilon}^{p}$ in the specimen is 1.2 .

\section{$\mathrm{V}=\mathbf{8} \mathrm{m} / \mathrm{s}$}

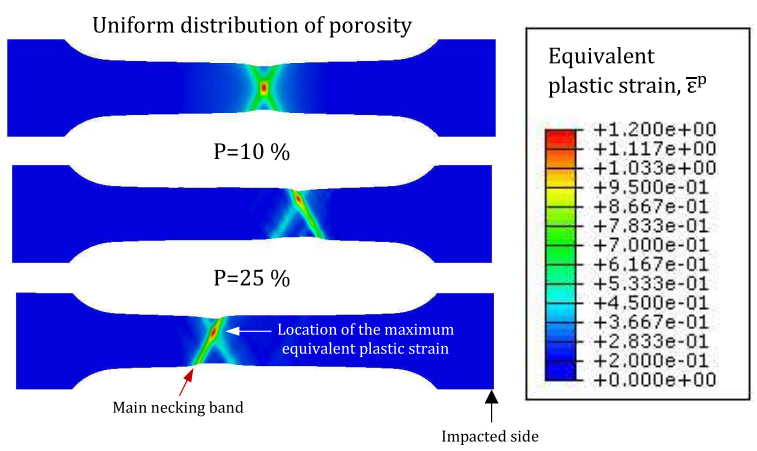

(a)

\section{$\mathrm{V}=20 \mathrm{~m} / \mathrm{s}$}

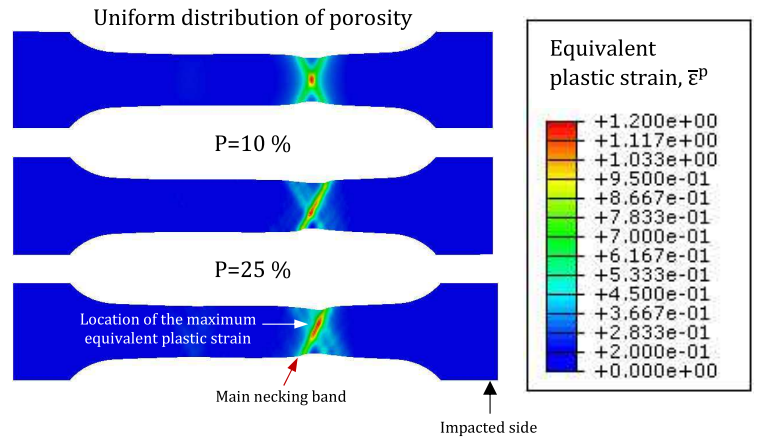

(b)

\section{$\mathrm{V}=\mathbf{2 5} \mathrm{m} / \mathrm{s}$}

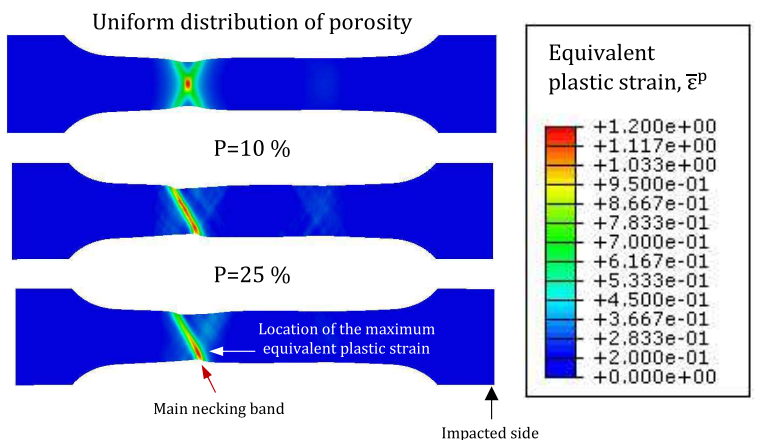

(c)

Figure 6: Contours of equivalent plastic strain $\bar{\varepsilon}^{p}$ for the material with uniform distribution of porosity, and the materials with non-uniform distributions of porosity corresponding to $P=10 \%$ and $P=25 \%$. Three impact velocities are considered: $8 \mathrm{~m} / \mathrm{s}$ in Fig. 6(a), $20 \mathrm{~m} / \mathrm{s}$ in Fig. 6(b), and $25 \mathrm{~m} / \mathrm{s}$ in Fig. 6(c). In all cases the loading time is such that the maximum value of $\bar{\varepsilon}^{p}$ in the specimen is 1.2 . 


\section{Experiments: steel AISI 430}

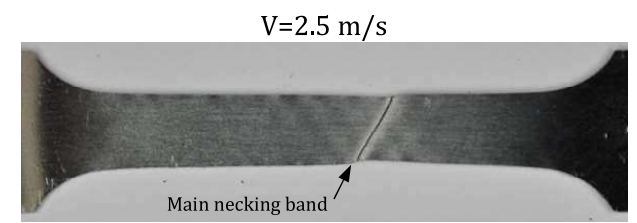

$\mathrm{V}=5 \mathrm{~m} / \mathrm{s}$

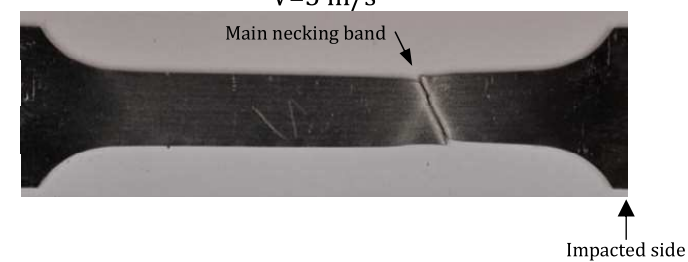

Figure 7: AISI 430 steel tensile samples tested by Vaz-Romero et al. [25] at $2.5 \mathrm{~m} / \mathrm{s}$ and $5 \mathrm{~m} / \mathrm{s}$ using a servo-hydraulic machine. The specimens show a fracture caused by a necking band aligned with one of the two directions of zero extension contained in the plane of the specimen. The location and orientation of the fracture depend on the applied velocity.

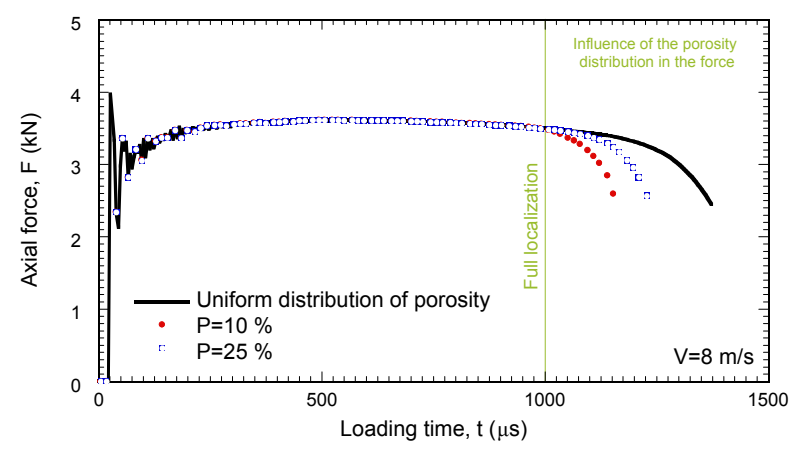

Figure 8: Axial force in the clamped side of the specimen $F$ as a function of the loading time $t$ for the material with uniform distribution of porosity, and the materials with non-uniform distributions of porosity corresponding to $P=10 \%$ and $P=25 \%$. The impact velocity is $V=8 \mathrm{~m} / \mathrm{s}$. show, in agreement with experiments, that the necking band which grows faster depends on the impact velocity and the specific distribution of porosity considered.

\section{Acknowledgements}

The research leading to these results has received funding from the European Union's Horizon2020 Programme (Excellent Science, Marie-Sklodowska-Curie Actions) under REA grant agreement 675602 (Project OUTCOME).

The authors acknowledge the fruitful discussions with Dr. Daniel Rittel (Technion).

\section{References}

[1] Aravas, N., 1987. On the numerical integration of a class of pressuredependent plasticity models. International Journal for Numerical Methods in Engineering 24, 1395-1416.

[2] Borsutzki, M., Cornette, D., Kuriyama, Y., Uenishi, A., Yan, B., Opbroek, E., 2005. Recommendations for dynamic tensile testing of sheet steels. Tech. rep., International Iron and Steel Institute (IISI).

[3] Clark, D. S., Duwez, P. E., 1948. Discussion of the forces acting in tension impact test of materials. Journal of Applied Mechanics 15, A243.

[4] Duwez, P. E., Clark, D., 1947. An experimental study of the propagation of plastic deformation under conditions of longitudinal impact. Proceedings - American Society for Testing Materials 47, 502-532.

[5] Grady, D. E., Benson, D. A., 1983. Fragmentation of metal rings by electromagnetic loading. Experimental Mechanics 12, 393-400.

[6] Gurson, A., 1977. Continuum theory of ductile rupture by void nucleation and growth. Part I: Yield criteria and flow rules for porous ductile media. ASME Journal of Engineering Materials and Technology 99, $2-15$.

[7] Hill, R., 1952. On discontinous plastic states, with special reference to localized necking in thin sheets. Journal of the Mechanics and Physics of Solids 1, 19-30.

[8] Hu, X., Daehn, G. S., 1996. Effect of velocity on flow localization in tension. Acta Materialia 44, 1021-1033.

[9] Hyun, H. C., Kim, M., Bang, S., Lee, H., 2002. On acquiring true stress-strain curves for sheet specimens using tensile test and FE analysis based on a local necking criterion. Journal of Materials Research 29, 695-707.

[10] Janiszewski, J., 2012. Ductility of selected metals under electromagnetic ring test loading conditions. International Journal of Solids and Structures 49, 1001-1008.

[11] Li, S., Liu, W. K., 2000. Numerical simulations of strain localization in inelastic solids using mesh-free methods. International Journal for Numerical Methods in Engineering 48, 1285-1309.

[12] Mann, H. C., 1936. High-velocity tension-impact tests. Proceedings American Society of Testing Materials 36, 85-109.

[13] Mann, H. C., 1937. Fundamental study of the design of impact test specimens. Proceedings American Society of Testing Materials 37, $102-118$.

[14] Mikkelsen, L., 1999. Necking in rectangular tensile bars approximated by a 2-D gradient dependent plasticity model. European Journal of Mechanics A/Solids 52, 805-818.

[15] Okazawa, S., 2010. Structural bifurcation for ductile necking localization. International Journal of Non-Linear Mechanics 45, 35-41.

[16] Okazawa, S., Usami, T., Noguchi, H., Fujii, F., 2002. Threedimensional necking bifurcation in tensile steel specimens. Journal of Engineering Mechanics 128, 479-486.

[17] Osovski, S., Rittel, D., Rodríguez-Martínez, J. A., Zaera, R., 2013. Dynamic tensile necking: Influence of specimen geometry and boundary conditions . Mechancis of Materials 62, 1-13.

[18] Rittel, D., Rotbaum, Y., Rodríguez-Martínez, J. A., Sory, D., Zaera, R., 2014. Dynamic necking of notched tensile bars: an experimental study. Experimental Mechanics 54, 1099-1109.

[19] Rusinek, A., Zaera, R., Klepaczko, J. R., Cheriguene, R., 2005. Analysis of inertia and scale effects on dynamic neck formation during tension of sheet steel. Acta Materialia 53, 5387-5400. 
[20] Simulia, 2013. ABAQUS/Explicit User's Manual, version 6.13 Edition. Dassault Systèmes, Providence, USA.

[21] Srivastava, A., Ponson, L., Osovski, S., Bouchaud, E., Tvergaard, V., Needleman, A., 2014. Effect of inclusion density on ductile fracture toughness and roughness. Journal of the Mechanics and Physics of Solids 63, 62-79.

[22] Tvergaard, V., 1981. Influence of voids on shear band instabilities under plane strain conditions. International Journal of Fracture 17 (4), 389407.

[23] Tvergaard, V., 1982. On localization in ductile materials containing spherical voids. International Journal of Fracture 18 (4), 237-252.

[24] Vaz-Romero, A., Rodríguez-Martínez, J. A., 2016. On the interplay between material flaws and dynamic necking. Mechanics Research Communications 72, 53-58.

[25] Vaz-Romero, A., Rodríguez-Martínez, J. A., Arias, A., 2015. The deterministic nature of the fracture location in the dynamic tensile testing of steel sheets. International Journal of Impact Engineering 86, 318335.

[26] Von Kármán, T., Duwez, P., 1950. The propagation of plastic deformation in solids. Journal of Applied Physics 21, 987.

[27] Wood, W. W., 1965. Experimental mechanics at velocity extremes very high strain rates. Experimental Mechanics 5, 361-371.

[28] Zhang, H., Ravi-Chandar, K., 2006. On the dynamics of necking and fragmentation - I. Real-time and post-mortem observations in $\mathrm{Al} 6061$ O. International Journal of Fracture 142, 183-217. 